\title{
On rectangles and countably generated families
}

\author{
by
}

\section{R. Daniel Mauldin (Gainesville, Fla)}

Abstract. This paper discusses some interrelationships between various statements involving. sets generated by rectangles, families of functions with a countable base as defined by Sierpiński, and some problems of Ulam. The existence of a $Q$-set and hence a normal, separable, nonmetrizable Moore space is obtained under some weak conditions. Also, the existence of a rarefied set of cardinality $c$ and Borel order $\omega_{1}$, is obtained under the assumption that every subset of $E^{2}$ is an $R_{\sigma \delta}$ set.

1. Introduction. Sierpiński has raised the following problem:

(S) For every family $F$ of real-valued functions defined on a set $X$ with $\operatorname{card}(F) \leqslant 2^{\aleph_{0}}$, does there exist a countable family $G$ of real-valued functions on $X$ such that each function in $F$ is the pointwise limit of a sequence from $G$ [18]?

Rothberger has shown that if $2^{\aleph_{0}}=\kappa_{1}$, then (S) holds [17].

It is shown here in Theorem 3.7 that (S) holds on every set $X$ with card $(X)$ $\geqslant 2^{N_{0}}$ if and only if

(F) every subset of $E^{2}$ is an $R_{\sigma \delta}$ set.

In statement $(\mathrm{F}), E^{2}$ is the plane and $R$ is the family of all generalized rectangles. in the plane: all sets of the form $A \times B$, where $A$ and $B$ are subsets of $E$.

The question of whether $(F)$ holds was raised by Ulam, Erdös and J. Johnson $[2,14,15]$.

In Theorem 4.1 it is shown that if $(F)$ holds then there is a rarefied number set $M$ with $\operatorname{card}(M)=2^{N_{0}}$ and such that $M$ has Baire (or Borel) order $\omega_{1}$.

In Theorem 4.4 it is shown that if $(F)$ holds and $B$ is a real normed linear space, $|B|=c=2^{\aleph_{0}}$, then there is a number set $M$ such that $B$ is embedded as a subspace of the Banach space of all bounded functions of Baire's class 1 on $M$.

In Theorem 4.5 it is shown that if $(F)$ holds and $2^{N_{0}}=2^{N_{1}}$, then there exists a $Q$-set and therefore a normal, separable nonmetrizable Moore space.

Also, generalizations of Sierpiński's problem on countably generated families are described and shown to be equivalent to certain generalizations of $(F)$ and in certain cases to another problem of Ulam's. 
Finally, it is shown in Theorem 5.1 that if $I$ is the unit interval, then Baire's class $\alpha$ on $I$ cannot be generated from the "inside" in $\gamma$ steps, where $\gamma<\alpha$. This implies a theorem of Dashiell's that the Banach spaces $B_{\alpha}(I)$ and $B_{\gamma}(I)$ are not isometric [4].

2. Notation and definitions. Let $X$ be a set and let $E$ denote the set of all real numbers. It is clear that statement (S) holds for a family $F$ of real-valued functions -defined on $X$ if and only if there is an algebra $A$ of bounded real-valued functions on $X$ which is separable under the uniform norm such that every function in $F$ is the pointwise limit of a sequence from $A$. Also, we may assume that $A$ contains the constant functions. In view of this, let us make the following definitions.

DefinITION 2.1. If $A$ is a subalgebra or sublattice of $E^{X}$ containing constants, then let $A_{0}=A$ and for each ordinal $\alpha$, let $A_{\alpha}$ be the family of all pointwise limits of sequences from the family $A_{\alpha-1}$, if $\alpha$ is not a limit ordinal and let $A_{\alpha}$ be the complete ordinary function system generated by $\bigcup_{\gamma<\alpha} A_{\gamma}$, if $\alpha$ is a limit ordinal $[7,12,13]$.

From this point on a separable subalgebra of $E^{X}$ will mean an algebra of bounded real-valued functions on $X$ which is separable under the uniform norm and which contains constants.

Definition 2.2. If $F$ is a subset of $E^{X}$ and $0<\alpha \leqslant \omega_{1}$, then $F$ will be said to have a countable base of order $\alpha$ provided there is a separable subalgebra $A$ of $E^{X}$ such that $F \subseteq A_{\alpha}$.

Of course, if a family $F$ has a countable base of order $\alpha$, then $\operatorname{card} F \leqslant 2^{\mathrm{N}_{0}}$.

Definition 2.3. If $G$ is a family of sets, let $M_{0}(G)=G$ and for each ordinal $\alpha, \alpha>0$, let $M_{\alpha}(G)$ be the family of all countable unions (intersections) of sets in $\bigcup M_{\gamma}(G)$, if $\alpha$ is odd (even) [2]. Limit ordinals are considered even.

Of course, $M_{\omega_{1}}(G)=M_{\omega_{1}+1}(G)=\bigcup_{\gamma<\omega_{1}} M_{\gamma}(G)$ and $M_{\omega_{1}}(G)$ is the smallest family of sets containing $G$ which is closed under countable unions and intersections.

Definition 2.4. If $G$ is a family of subsets of $X$, then let $Z_{0}(G)$ be $G, C Z_{0}(G)$ be the family of all complements of sets in $G$ and for each ordinal $\alpha$, let $Z_{\alpha}(G)$ be the family of all countable intersections of sets from $\bigcup_{\gamma<\alpha} C Z_{\gamma}(G)$ and let $C Z_{\alpha}(G)$ be the family of all complements of sets in $Z_{\alpha}(G)$ [12].

We have $Z_{\omega_{1}}(G)=C Z_{\omega_{1}}(G)=\bigcup_{\gamma<\omega_{1}} Z_{\gamma}(G)$ and that $Z_{\omega_{1}}(G)$ is the $\sigma$-field (algebra) of sets generated by $G$.

The family of all sets of the form $A \times B$, where $A$ and $B$ are subsets of the real line, $E$, will be denoted by $R$. Thus, $R$ is the family of all generalized rectangles in the plane, $E^{2}$.

3. Countably generated families and generalized rectangles. In [2], it is shown that if every subset of $E^{2}$ is in $M_{\omega_{1}}(R)$, then there is a countable ordinal $\alpha$ such that (*) $P\left(E^{2}\right)=M_{\alpha}(R)$, where $P\left(E^{2}\right)$ denotes the power set of $E^{2}$. In this section, we develop a functional equivalence to $(*)$ in terms of families which have a countable base.

LEMMA 3.1. Let $H$ be a family of subsets of $X$ and let $0<\alpha \leqslant \omega_{1}$. The following two statements are equivalent:

1) there is a countable family, $G$, of subsets of $X$ such that $H \subseteq M_{\alpha}(G)$, and

2) there is a countable field, $W$, of subsets of $X$ such that $H \subseteq Z_{\alpha}(W)\left(H \subseteq C Z_{\alpha}(W)\right)$, if $\alpha$ is even $(o d d)$.

This lemma follows immediately from the facts that if $G$ is a countable family of sets, then the field generated by $G$ is countable and if $W$ is a field, then $C Z_{\alpha}(W)$ $\supseteq M_{\alpha}(W)$, if $\alpha$ is odd and $Z_{\alpha}(W) \supseteq M_{\alpha}(W)$, if $\alpha$ is even.

LEMMA 3.2. Let $\Phi$ be a vector lattice of bounded real-valued functions defined on a set $X$ which contains constants, let $T=\left\{f^{-1}(K) \mid f \in \Phi\right.$ and $K$ is closed $\}$ and let $0<\alpha \leqslant \omega_{1}$. Suppose $g$ is a real-valued function defined on $X$. Then the following two statements are equivalent:

1) the function $g$ is in the family $\Phi_{\alpha}$, and

2) $g^{-1}(K) \in Z_{\alpha}(T)$, for each closed set $K$.

Moreover, if $A$ is the closure of $\Phi$ under the uniform norm, then $A$ is a subalgebra of $E^{X}$ and for each $\alpha>0, A_{\alpha}=\Phi_{\alpha}$.

This is Theorem 3.5 of [13].

LEMMA 3.3. Let $W$ be a countable field of subsets of $X$ and let

$$
\Phi=\left\{\sum_{i=1}^{n} a_{i} \xi_{A_{l}} \mid a_{i} \in E \text { and } A_{i} \in W\right\} .
$$

Let $A$ be the closure under the uniform norm of $\Phi$. Then $A$ is a separable subalgebra of $E^{\mathrm{X}}$ and for each $\alpha>0$, a function $f$ is in the family $A_{\alpha}$ if and only if $f^{-1}(K) \in Z_{\alpha}(W)$, for each closed set $K$.

Lemma 3.3 follows from the preceding lemma upon noticing that $\Phi$ is a vector lattice of bounded functions on $X, \Phi$ contains constants and the countable set $D$ consisting of all functions in $\Phi$ which map into the rational numbers is dense in $A$.

LEMMA 3.4. Let $M$ be a bounded number set and let $\Phi$ be the family of all realvalued functions which are uniformly continuous on $M$. Then $\Phi$ is a separable subalgebra of $E^{M}$ and $\Phi_{\alpha}=C_{\alpha}$, for each ordinal $\alpha, 0<\alpha \leqslant \omega_{1}$, where $C$ is the family of all bounded real-valued continuous functions on $M$.

Proof. Since $\Phi$ is the family of all restrictions of real-valued continuous functions defined on $\bar{M}$, the closure of $M$, it follows that $\Phi$ is a separable subalgebra of $E^{M}$.

We now show that $\Phi_{1}$ is the family of all functions in Baire's class 1 on $M$.

First assume $f$ is a bounded continuous function defined on $M$. 
For each rational $q$, with $q \leqslant\|f\|$, let $U_{q}=\{x \mid f(x)<q\}$, let $0_{q}$ be an open subset of $\bar{M}$ such that $0_{q} \cap M=U_{q}$ and let

$$
h_{q}(x)=\left\{\begin{array}{lll}
q, & \text { if } & x \in 0_{q}, \\
\|f\|, & \text { if } & x \in \bar{M}-0_{q} .
\end{array}\right.
$$

Then, for each such rational $q, h_{q}$ is upper semi continuous on $\bar{M}$. Let $\left\{g_{n q}\right\}_{n \neq 1}^{\infty}$ be a decreasing sequence of continuous functions on $\bar{M}$ which converges to $h_{q}$. Let $f_{n q}$ be the restriction of $g_{n q}$ to $M$.

We have $f_{n q} \geqslant f$ and $f_{n q} \in \Phi$, for each $n$ and $q$. Assume $x \in M, \varepsilon>0$ and $q$ is a rational such that $q<f(x)+\varepsilon \leqslant\|f\|$. Then there is an $n$ such that $q<f_{n q}(x)<f(x)+\varepsilon$. Thus, $f$ is the infinum of a countable subfamily of $\Phi$ and since $\Phi$ is a lattice, $f$ is the limit of a decreasing sequence from $\Phi$.

Therefore, in the terminology of [13], $f \in$ US $\Phi$. Thus, $C \subset$ US $\Phi$ and USC $\subset \mathrm{US}(\mathrm{US} \Phi)=\mathrm{US} \Phi \subset \mathrm{USC}$.

Thus, US $\Phi=$ USC and similarly LS $\Phi=$ LSC. Also, US $(\operatorname{LS} \Phi)=$ US $(\operatorname{LSC})$ and $\operatorname{LS}(\mathrm{USS} \Phi)=\mathrm{US}(\mathrm{LSC})$. Since a function is in Baire's class 1 on $M$ if and only if it is in the intersection of the classes US(LSC) and LS(USC) ([13], Th. 3.5) and $\Phi_{1}=\operatorname{US}(\operatorname{LS} \Phi) \cap \operatorname{LS}(\operatorname{US} \Phi)$ ([13], Th. 3.5), we have $\Phi_{1}$ is the family of all functions of Baire's class 1 on $M$.

If follows by transfinite induction that $\Phi_{\alpha}=C_{\alpha}$, for each ordinal $\alpha, 0<\alpha \leqslant \omega_{1}$.

LEMMA 3.5. Let $A$ be a separable subalgebra of $E^{X}$ and let $T=\left\{f^{-1}(K) \mid f \in A\right.$ and $K$ is closed $\}$. Then there is a countable subfamily $G_{1}$ of $C T$ such that $C T \subseteq\left(G_{1}\right)_{\sigma}$ and there is a countable subfamily $G_{2}$ of $T$ such that $T \subseteq\left(G_{2}\right)_{8}$.

Proof. Let $\left\{f_{n}\right\}_{n=1}^{\infty}$ be a countable dense subset of $A$ and let $G_{1}$ be the family of all sets of the form $f_{n}^{-1}(I)$, where $n$ is a positive integer and $I$ is an open interval with rational endpoints.

It $Z \in C T$, then $Z=f^{-1}(U)$, for some $f \in A$ and some open set $U$. Assume $f(s) \in U$. Let $n$ be a positive integer and $p$ and $q$ be rational numbers such that $p<f(s)<q$ and the open interval $(p-1 / n, q+1 / n)$ is a subset of $U$. Let $k$ be a positive integer such that $\left\|f-f_{k}\right\|<1 / n$. Then $p-1 / n<f_{k}(s)<q+1 / n$ and $s \in f_{k}^{-1}(p-1 / n$, $q=1 / n)$. It follows that $C T \subseteq\left(G_{1}\right)_{\sigma}$. Also, by considering complements it follows that the family $G_{2}=C G_{1}$ is a countable subfamily of $T$ and that $T \subseteq\left(G_{2}\right)_{\delta}$.

THEOREM 3.6. Let $F$ be a subfamily of $E^{X}$ having cardinality $2^{\mathrm{N}_{0}}$, let $H=\left\{f^{-1}(K) \mid f \in F\right.$ and $K$ is closed). The following two statements are equivalent:

1) $F$ has a countable base of order 1 ,

2) there is a countable family $G$ of subsets of $S$ such that $M_{2}(G) \supseteq H$.

Proof. First assume $A$ is a separable subalgebra of $E^{X}$ such that $F \subseteq A_{1}$. We may assume $A$ is a Banach space. Then $A$ is also a vector lattice of real-valued functions on $X$ and it follows from Lemma 3.2 that a function $g$ is in $A_{1}$ if and only if $g^{-1}(K) \in Z_{1}(T)$, for each closed set $K$, where $T=\left\{f^{-1}(K) \mid f \in A\right.$ and $K$
is closed $\}$.

By Lemma 3.5, there is a countable subfamily $G$ of $C T$ such that $G_{\sigma} \supseteq C T$. Then $M_{2}(G)=G_{\sigma \delta} \supseteq Z_{1}(T)=(C T)_{\delta}$. Thus, $M_{2}(G) \supseteq H$.
Now, assume that there is a countable family $G$ of subsets of $X$ such that $M_{2}(G) \supseteq H$. We shall also assume that the family of functions $F$ separates points of $X$. It is easy to check that there is no loss in generality by factoring out the "stationary" sets of $F$.

Let $W=\left\{A_{p}\right\}_{p=1}^{\infty}$ be a countable field of subsets of $X$ such that $H \subseteq Z_{2}(W)$ (Lemma 3.1).

Let $\varphi(x)=2 \sum_{p=1}^{\infty} 3^{-p} \xi_{A_{p}}(x)$, for each $x$ in $X$ where $\xi_{A}$ is the characteristic function of a set $A$. The function $\varphi$ has been termed the characteristic function of a sequence of sets $\left\{A_{p}\right\}_{p=1}^{\infty}$. Then $\varphi^{-1}$ establishes a biunivocal correspondence between the classes $Z_{2}(W)=W_{a \delta}$ and the family of all $G_{\delta}$ or inner limiting subsets of the image $M$ of $X$ under $\varphi$ ([19], p. 212). Also, since we are assuming that the family $F$ separates points of $X$, it follows that if $x$ and $y$ are points of $X$, then there must be some member $A_{p}$ of $W$ such that $x \in A_{p}$ and $y \in A_{p}^{\prime}$. Therefore, the function $\varphi$ is 1 to 1 ([19], p. 215).

Thus, if $f \in F$, then $f \circ \varphi^{-1}$ is of Baire's class 1 on $M$, since

$$
(f \circ \varphi)^{-1}(K)=\varphi\left(f^{-1}(K)\right) \text { and } \varphi\left(f^{-1}(K)\right)
$$

is a $G_{\delta}$ subset of $M([13]$, Th. 3.5). Let $\Phi$ be the family of all uniformly continuous functions on $M$. If $f \in F$, then by Lemma $3.4, f \circ \varphi^{-1} \in \Phi$, and thus $f \in A_{1}$, where $A=\{f \circ \varphi \mid f \in \Phi\}$. Of course, $A$ is a separable subalgebra of $E^{X}$.

This completes the argument for Theorem 3.6.

We shall now show the equivalence of statements $(S)$ and $(F)$.

THEOREM 3.7. Each two of the following statements are equivalent:

1. (S) each family of $2^{\aleph_{0}}$ real-valued functions defined on $E$ has a countable base of order 1 [18],

2. for each family $H$ of $2^{\aleph_{0}}$ subsets of $E$ there exists a countable family $G$ such that $M_{2}(G) \supseteq H$,

3. $(F): P\left(E^{2}\right)=M_{2}(R)$.

Proof. The proof that statements 2 and 3 are equivalent is contained in [2]. Theorem 3.6 shows that statement 2 implies statement 1 . Finally, given a family $H$ of $2^{\aleph_{0}}$ subsets of $E$, let $F$ be the family of all characteristic functions of members of $H$, Theorem 3.6 shows that if statement 1 holds, then there is a countable family $G$ such that $M_{2}(G) \supseteq H$. Thus, statement 1 implies statement 2. Q.E.D.

In [2], it is shown that if $P\left(E^{2}\right)=M_{\omega_{1}}(R)$, then there is some countable ordinal $\alpha$ such that $P\left(E^{2}\right)=M_{\alpha}(R)$. Although there seem, to be no known models in which $P\left(E^{2}\right)=M_{\alpha}(R)$, for some $\alpha>2$, some equivalences to this proposition are now developed and a functional equivalence to the solvability of another problem of Ulam is given. (See Addendum.)

THEOREM 3.8. Let $F$ be a subfamily of $E^{X}$, let $H=\left\{f^{-1}(K) \mid f \in F\right.$ and $K$ is closed $\}$ and let $J=\left\{f^{-1}(U) \mid f \in F\right.$ and $U$ is open $\}$ and let $\alpha$ be an ordinal, $0<\alpha \leqslant \omega_{1}$. 
Tihen $F$ has a countable base of order $\alpha$ if and only if there is a countable family $G$ of subsets of $X$ such that the appropriate condition below holds:

a) if $\alpha$ if finite and odd, $H \subseteq M_{\alpha+1}(G)$,

b) if $\alpha$ is finite and even, $J \subseteq M_{\alpha+1}(G)$,

c) if $\alpha$ if infinite and even, $H \subseteq M_{\alpha}(G)$,

d) if $\alpha$ if infinite and odd, $J \subseteq M_{\alpha}(G)$.

Proof. First, assume $F$ has a countable base of order $\alpha, A$. Let $T=\left\{f^{-1}(K) \mid\right.$ $f \in A$ and $K$ is closed\}. By Lemma 3.2, a function $g$ is in $A_{\alpha}$ if and only if $g^{-1}(K)$ $\in Z_{\alpha}(T)$, for every closed set $K$ or, considering complements, $g^{-1}(U) \in C Z_{\alpha}(T)$, for every open set $U$.

Let $G$ be the family $G_{1}$ as described in Lemma 3.5. It follows by transfinite induction that $M_{\alpha+1}(G) \supseteq Z_{\alpha}(T)$ if $\alpha$ is finite and odd, $M_{\alpha+1}(G) \supseteq C Z_{\alpha}(T)$, if $\alpha$ is finite and even. Also, if $\alpha$ is infinite and even, $Z_{\alpha}(T) \subseteq M_{\alpha}(G)$ and if $\alpha$ is infinite arid odd, $C Z_{\alpha}(T) \subseteq M_{\alpha}(G)$.

It follows via Lemma 3.2 that the appropriate condition a), b), c) or d) holds.

Now, assume that there is a countable family $G$ such that the families $H$ or $J$ are generated in the required number of steps.

Let $W=\left\{A_{p}\right\}_{p=1}^{\infty}$ be the countable field generated by $G$ and let $\varphi$ be the characteristic function of the sequence $\left\{A_{p}\right\}_{p=1}^{\infty}$. Again, it is assumed that the family $F$ separates points of $X$ and consequently, as in Theorem $3.7, \varphi$ is 1 to 1 .

Let $M=\varphi(X)$ and let $K$ be the family of all closed sets with respect to $M$.

It follows from the properties of the characteristic function that $E \in M_{\alpha+1}(W)$ if and only if $\varphi(E) \in Z_{\dot{\alpha}}(K)$, if $\alpha$ is finite and even or $\varphi(E) \in C Z_{\alpha}(K)$, if $\alpha$ is finite and odd. Also, if $\alpha$ is infinite, then $E \in M_{\alpha}(W)$ if and only if $\varphi(E) \in Z_{\alpha}(K)\left(C Z_{\alpha}(K)\right)$ for $\alpha$ even (odd). (See [19], p. 212.)

Let $\Phi$ be the family of all uniformly continuous functions on $M$. Let $A$ $=\{f \circ \varphi \mid f \in \Phi\}$. Then $A$ is a separable subalgebra of $E^{X}$.

Suppose $\alpha$ is finite and odd. If $f \in F$, then $f^{-1}(F) \in M_{\alpha+1}(G) \subseteq M_{\alpha+1}(W)$ for every closed set $F$. Therefore, $\left(f \circ \varphi^{-1}\right)^{-1}(F)=\varphi\left(f^{-1}(F)\right) \in Z_{\alpha}(K)$, for every closed set $F$ and $f \circ \varphi^{-1}$ is of Baire class $\alpha$ on $M$. Thus, $f \circ \varphi^{-1} \in \Phi_{\alpha}$ and $f \in A_{\alpha}$. The argument for the other cases is similar.

THEOREM 3.9. Let $\alpha$ be an ordinal, $0<\alpha<\omega_{1}$. Then

a) if $0<\alpha<\omega_{0}$, each two of the following statements are equivalent:

i) each family of $2^{N_{0}}$ real-valued functions on $E$ has a countable base of order $\alpha$

ii) for each family $H$ of $2^{N_{0}}$ subsets of $E$, there is a countable family $G$ of subsets of $E$ such that $M_{\alpha+1}(G) \supseteq H$

iii). $P\left(E^{2}\right)=M_{\alpha+1}(R)$.

b) if $\omega_{0} \leqslant \alpha<\omega_{1}$, then each two of the following statements are equivalent:

i) each family of $2^{\aleph_{0}}$ real-valued functions on $E$ has a countable base of order ' $\alpha$,

ii) for each family $H$ of $2^{\aleph_{0}}$ subsets of $E$ there is a countable family $G$ such that $M_{\alpha}(G) \supseteq H$,

iii) $P\left(E^{2}\right)=M_{\alpha}(R)$.
Proof. Theorem 8 of [2] shows that (ii) and (iii) of (a) and (b) are equivalent. Theorem 3.9 shows that (ii) implies (i) in case (a) or (b).

In order to show that statement (i) implies (ii), let $H$ be a family of $2^{\text {No }_{0}}$ subsets of $E$ and let $F$ be the family of all characteristic functions of members of $M$. If $\xi_{A} \in F$, then the inverse image under $\xi_{A}$ of the open interval $\left(\frac{1}{2}, \frac{3}{2}\right)$ is the inverse image under $\xi_{A}$ of the closed interval $\left[\frac{1}{2}, \frac{3}{2}\right]$ and thus Theorem 3.9 shows that (i). implies (ii) of (a) or (b).

Ulam has asked whether statement 2 in Theorem 3.10 below holds in [22] and [17], p. 405. We give a functional equivalent of this problem in statement 1 of Theorem 3.10 .

THEOREM 3.10. The following two statements are equivalent:

1) each family $F$ of $2^{N_{0}}$ real-valued functions defined on $E$ has a countable base of order $\omega_{1}$,

2) for each family $H$ of $2^{N_{0}}$ subsets of $E$ there is a countable family $G$ of. subsets of $E$ such that $H \subseteq M_{\omega_{1}}(G)$.

The proof of this theorem proceeds exactly as in the earlier theorems and is not given. It should be noted that it is unknown whether statement 2 of Theorem 3.10 implies $P\left(E^{2}\right)=M_{\omega_{1}}(R)$ [2].

\section{Some applications of $P\left(E^{2}\right)=M_{2}(R)$.}

THEOREM 4.1. If $P\left(E^{2}\right)=M_{2}(R)$, then there is a number set $M$ of cardinality $2^{N_{0}}$ which is rarefied (or has property $\lambda,[10], \mathrm{p} .517$ ). Moreover, $M$ may be taken to have Baire or Borel order $\omega_{1}$ : there exist Borel sets with respect to $M$ of arbitrarily high class.

Proof. Let $H$ be the family of all Borel subsets of $E$; card $H=2^{\text {No }}$. Therefore, by Theorem 3.7 and Lemma 3.1 , there is a countable field $W=\left\{A_{p}\right\}_{p=1}^{\infty}$ such that $H \subseteq W_{\sigma \delta}$. Let $\varphi$ be the characteristic function of the sequence $\left\{A_{p}\right\}_{p=1}^{\infty}$ and let $M=\varphi(E)$. It follows from Section 4.3 (ii) (p. 220) of [19], that the set $M$ is rarefied. Finally, it was shown in [2] (Theorem 12) that the family $W$ has Borel order $\omega_{1}$. Since $\varphi^{-1}$ defines an isomorphism of the Borel classes of $M$ with those generated by $W$, it follows that $M$ has Borel order $\omega_{1}$. Q.E.D.

It seems strange that there is a separable metric space in which every countable set is a $G_{\delta}$ and yet the space has Borel order $\omega_{1}$.

Rarefied spaces have been studied by F. Burton Jones, Kuratowski, S. Besicovitch and others. It seems that all previously known examples have had cardinality $\kappa_{1}$ and are of unknown Borel order ([19], p. 219).

Let $C$ be the family of all generalized cubes; $C$ is the family of all sets $A \times$ $\times B \times C$, where $A, B$ and $C$ are subsets of $E$.

THEOREM 4.2. The following two statements are equivalent:

1) the graph of every real-valued function defined on $E^{2}$ is in $M_{2}(C)$, and

2) $P\left(E^{2}\right)=R_{\sigma \delta}=M_{2}(R)$ 
Proof. First, assume statement 1 holds. Let $A$ be a subset of $E^{2}$. Then by assumption the graph of the characteristic function of $A, \xi_{A}$, is in the family $C_{\alpha \delta}$ $=M_{2}(C)$. It can be shown by transfinite induction that if $J \in M_{\alpha}(C)$ and $Q$ is a plane parallel to the $x y$-plane then $Q \cap J$ is in the class $M_{\alpha}(R)$. Thus, $A$ is in the family $M_{\alpha}(R)$ and statement 2 holds.

Now assume statement 2 and let $f$ be a function from $E^{2}$ into $E$. Lel

$(*)$

$$
\begin{aligned}
D_{n}= & f^{-1}((-\infty,-n]) \times(-\infty, n] \cup \\
& \cup \bigcup_{p}\left[f^{-1}\left(\left(-n+\frac{p}{n \cdot 2^{n}},-n+\frac{p+1}{n \cdot 2^{r}}\right)\right) \times\left(-n+\frac{p}{n \cdot 2^{n}},-n+\frac{p+1}{n \cdot 2^{n}}\right)\right] \cup \\
& \cup f^{-1}([n,+\infty)) \times[n,+\infty),
\end{aligned}
$$

where $p$ ranges over the integers from 0 to $2 n^{2} \cdot 2^{n}-1$.

For each $n$, the graph of $f$ is a subset of $D_{n}$. In fact, $\bigcap_{n=1}^{\infty} D_{n}$ is the graph of $f$. It can be shown by transfinite induction that if $J \in M_{\alpha}(R)$ and $A \subseteq E$, then $J \times A$ . $\in M_{\alpha}(C)$. Thus, each of the sets appearing on the right hand side of (*) is in the family $M_{2}(C)$. Since $M_{2}(C)$ is closed under finite unions, we have that for each $n, D_{n} \in M_{2}(C)=C_{\sigma \delta}$. Since $M_{2}(C)$ is closed under countable intersections, the graph of $f$ is in $M_{2}(C)$. Q.E.D.

If $A \in M_{2}(C)$, then there is a countable subfamily $\left\{A_{n} \times B_{n} \times C_{n}\right\}_{n=1}^{\infty}=W$ such that $A \in W_{\sigma \delta}$. Thus, as a corollary of Theorem 4.2 , we have a solution to a problem of Ulam in the following:

Corollary 4.3. Suppose $P\left(E^{2}\right)=R_{\sigma \delta}$. If $G$ is a group defined on $E$, then there is a countable sequence of number sets such that the "representation" of $G$ would be of class 2 in the terminology of Ulam ([22], p. 7).

THEOREM 4.4. Suppose $P\left(E^{2}\right)=R_{\sigma \delta}$. If $B$ is a real normed linear space having cardinality $2^{\mathrm{N}_{0}}$, then there is a subset $M$ of the Cantor set such that $B$ is linearly isometric to a subspace of the Banach space of all bounded functions of Baire's class 1
on $M$.

Proof. Let $S$ be the unit ball of the dual of $B$ and for each $b \in B$, let $h_{b}\left(b^{*}\right)$ $=b^{*}(b)$, for each $b^{*} \in S$. This defines a linear isometry of $B$ with the space $H$ of
all functions $h_{b}$ under the uniform norm.

Let $A$ be a separable subalgebra of $E^{S}$ such that $H \subseteq A_{1}$. Let $\left\{A_{n}\right\}_{n=1}^{\infty}$ be the sequence of sets, $G_{1}$, described in Lemma 3.5 and let $\varphi$ be the characteristic function of the sequence $\left\{A_{n}\right\}_{n=1}^{\infty}$. Then, for each $f \in A, f \circ \varphi^{-1}$ is a continuous function on the subset $M=\varphi(S)$ of the Cantor set. Therefore, the transformation $T(h)$ $=h \circ \varphi^{-1}$ describes a linear isometry of $H$ with a subspace of $B_{1}(M)$, the Banach space of all bounded functions of Baire's class 1 on $M$ under the uniform norm.
The theorem now follows.
THEOREM 4.5. Suppose $P\left(E^{2}\right)=R_{\sigma \delta}$ and $2^{N_{0}}=2^{\aleph_{1}}$. Then there exists a $Q$-set $[16]$ and therefore there is a normal, separable, nonmetrizable Moore space [1].

Proof. Let $T$ be a number set with $\operatorname{card}(T)=\aleph_{1}$. Let $H$ be the family of all subsets of $T$. Then $\operatorname{card} H=2^{\mathrm{N}_{1}}=2^{\mathrm{N}_{0}}$. Therefore, by Theorem $8 \mathrm{~b}$ of [2], there is a countable family $G$ of subsets of $T$ such that $H \subseteq M_{\alpha}(G)$. Let $W=\left\{A_{p}\right\}_{n=1}^{\infty}$ be the countable field of subsets of $T$ generated by $G$ (complements are taken with respect to $T$.) Let $\varphi$ be the characteristic function of $\left\{A_{p}\right\}_{p=1}^{\infty}$ and let $M$ be $\varphi(T)$.

Since $W$ separates points of $T, \varphi$ is $1-1$ and therefore card $M=\kappa_{1}$ ([18], p. 215). If $K \subseteq M$, then $\varphi^{-1}(K) \in H$ and therefore $\varphi^{-1}(K)$ is a $W_{\sigma \delta}$ set. Then, $K=\varphi\left(\varphi^{-1}(K)\right)$ is a $G_{\delta}$ subset of $M([17]$, p. 212) and ([17], p. 217). Therefore, $M$ is a $Q$-set. Q.E.D.

R. W. Heath has shown that the existence of a $Q$-set is equivalent to the existence of a normal separable, nonmetrizable Moore space [7]. Thus, under the hypothesis of Theorem 4.5, the answer to F. Burton Jones' normal Moore space problem is no.

QUESTION. If there is a $Q$-set, is every subset of $E$ of cardinality $\wedge_{1}$ a $Q$-set?

Martin and Solovay have shown in [10] that Martin's Axiom together with the negation of $C H$ implies Lusin's second continuum hypothesis: $2^{\aleph_{0}}=2^{\aleph_{1}}$. Also, Kunen has shown in [8] that Martin's Axiom implies $P\left(E^{2}\right)=R_{\sigma \delta}$. Thus, from these results and Theorem 4.5 we have a result of Tall and Silver [19]:

THEOREM 4.6. The existence of a normal, separable, nonmetrizable Moore space (or Q-set) is consistent with Zermelo-Frankel + Axiom of Choice.

Recently, Kunen and Tall have given a model of ZFC in which Martin's Axiom fails, but $2^{N_{0}}=2^{N_{1}}$ and $R_{\sigma \delta}=P\left(E^{2}\right)$.

5. Inner bases. Let $F$ be a family of real-valued functions defined on a set $X$. $F$ will be said to have an inner base provided $F$ has a countable base, $A$, of order $\alpha$ for some $\alpha, 0<\alpha \leqslant \omega_{1}$ such that $A \subseteq F$. The smallest such ordinal $\alpha$ will be called the inner order of $F$.

As an example, consider the Banach space, $B_{\alpha}(I)$, consisting of all bounded functions of Baire's class $\alpha$ on the unit interval $I$. Then, $B_{\alpha}(I)$ has an inner base of order $\alpha$, namely, any countable and dense subset of $C$, the space of all continuous functions on $I$. It is shown below that $\alpha$ is the inner order of $B_{\alpha}(I)$, for each ordinal $\alpha, 0<\alpha \leqslant \omega_{1}$ and that this is an isometric invariant among these spaces.

The problem of determining when a family $F$ has an inner base seems difficult.

THEOREM 5.1. Suppose $0<\gamma<\alpha$. The space $B_{\alpha}(I)$ does not have an inner base of order $\gamma$.

Proof. Assume that $A$ is an inner base of $B_{\alpha}(I)$ of order $\gamma$. Let $Z_{0}$ be the family of all zero sets of functions in $A$.

We have that $f \in A_{\delta}, \delta>0$, if and only if $f^{-1}(K) \in Z_{\delta}$ for every closed set $K$ ([13], Th. 3.5). 
Let $G_{2}$ be a countable subfamily of $C Z_{0}$ such that $C Z_{0} \subseteq\left(G_{2}\right)_{\sigma}$ (Lemma 3.5). Let $W$ be the countable field generated by $G_{2}, W=\left\{A_{p}\right\}_{p=1}^{\infty}$. Let $\varphi$ be the characteristic function of $W$ :

$$
\varphi(x)=\sum_{p=1}^{\infty} \frac{2}{3^{p}} \xi_{A_{p}}(x) .
$$

Let $M$ be the image of $I$ under $\varphi: M=\varphi(I)$. Since $\varphi$ is 1 to $1, M$ is a Borel set. If $\xi_{K} \in B_{\alpha}(I)$, then $\xi_{K} \in A_{\gamma}$ and thus $\xi_{K} \circ \varphi^{-1}$ is in Baire class $\gamma$ on $M$. Thus, $\varphi(K)$ is a multiplicative class $\gamma$ with respect to $M$.

Of course, the graph of $\varphi$ is a Borel subset of $I \times I$ and therefore contains a perfect set $H$. Let $T$ be a subset of $H$ of exactly multiplicative class $\alpha$. Since the $x$-projection mapping of the graph of $\varphi$ to $I, \pi_{x}$, is a homeomorphism when restricted to $H$, we have $\pi_{x}(T)$ is of exactly multiplicative class $\alpha$ on $I$. Similarly, $\pi_{y}(T)$ is of exactly multiplicative class $\alpha$ on $M$. Therefore $\varphi\left(\pi_{x}(T)\right)=\pi_{y}(T)$ is not a multiplicative class $\gamma$ on $M$. This contradiction proves the theorem.

THEOREM 5.2. Suppose $0 \leqslant \gamma<\alpha \leqslant \omega_{1}$. There does not exist a continuous transformation of $B_{\gamma}(I)$ onto $B_{\alpha}(I)$, which preserves pointwise limits.

Proof. Suppose $0 \leqslant \gamma<\alpha \leqslant \omega_{1}$ and $T$ is a continuous transformation of $B_{\gamma}$ onto $B_{\alpha}$ which preserves pointwise limits.

Of course, $\gamma>0$, since $C(I)=B_{0}(I)$ is separable while $B_{\alpha}(I), \alpha>0$ is not separable.

Let $A$ be the subalgebra of $B_{\alpha}(I)$ generated by $T(C)=T\left(B_{0}(I)\right)$ which contains constants. Then $A$ is a separable subalgebra of $B_{\alpha}(I)$ and since $T$ preserves pointwise limits it follows that $A_{\gamma} \supseteq T\left(B_{\gamma}(I)\right)=B_{\alpha}(I)$.

This contradicts Theorem 5.1 and proves the theorem.

Theorem 5.2 implies the following theorem of Dashiell [3].

THEOREM 5.3. Suppose $0 \leqslant \gamma<\alpha \leqslant \omega_{1}$. The Banach spaces $B_{\alpha}(I)$ and $B_{\gamma}(I)$ are not linearly isometric.

Proof. Suppose $T$ is a linear isometry of $B_{\gamma}(I)$ onto $B_{\alpha}(I)$. Let $X_{\gamma}(I)$ and $X_{\alpha}(I)$ be the "Stone-Cech compactifications of $B_{\gamma}(I)$ and $B_{\alpha}(I)$, respectively $[6,13]$. Thus, there is a 1-1 mapping, $\varphi_{\gamma}$, of $I$ onto a dense subset, $D_{\gamma}$, of $X_{\gamma}(I)$ such that the function $f \circ \varphi_{\gamma}^{-1}$ has an extension to a continuous function $\hat{f}$ on $X_{\gamma}(I)$, for each $f \in B_{\gamma}(I)$ and the mapping $f \rightarrow \hat{f}$ is a linear isometry of $B_{\gamma}(I)$ with $C\left(X_{\gamma}(I)\right)$. Similar statements hold concerning $B_{\alpha}(I)$ and $C\left(X_{\alpha}(I)\right)$.

We assume $\gamma>0$. Since $B_{0}(I)=C(I)$ is the only separable space among the spaces $B_{\alpha}(I), 0 \leqslant \alpha \leqslant \omega_{1}$.

It follows that $C\left(X_{\gamma}(I)\right)$ and $C\left(X_{\alpha}(I)\right)$ are linearly isometric. Thus, there is a homeomorphism $\varphi$ from $X_{\alpha}(I)$ onto $X_{\gamma}(I)$ and a function $p$ in $C\left(X_{\alpha}(I)\right)$ with $|p(x)|=1$ for all $x$ in $X_{\alpha}(I)$ such that $(T(g))(x)=p(x) g(\varphi(x))$, for all $x \in X_{\alpha}(I)$ and all $g$ in $C\left(X_{\gamma}(I)\right)$ ([6], p. 442).

If $r \in I$, then $\xi_{(r)} \in B_{\gamma}(I)$ and the extension of $\xi_{(r)}$ to $X_{\gamma}(I)$ is the characteristic function of $\varphi_{\gamma}(r)$ on $X_{\gamma}(I)$.
Therefore, $T\left(\xi_{(r)}\right)=p\left(\varphi^{-1}\left(\varphi_{\gamma}(r)\right)\right) \xi_{(p-1}{ }^{-1}\left(\varphi_{\gamma}(r)\right)$. It follows that $\varphi^{-1}\left(\varphi_{\gamma}(r)\right) \in D_{\alpha}$, since otherwise the zero function would have two extensions to continuous functions on $X_{\alpha}(I)$. Thus, $\varphi^{-1}\left(\varphi_{\gamma}(I)\right) \subseteq \varphi_{\alpha}(I)$. Similarly we have $\varphi\left(\varphi_{\alpha}(I)\right) \subseteq \varphi_{\gamma}(I)$.

Then $\Psi=\varphi_{\alpha}^{-1} \circ \varphi^{-1} \circ \varphi_{\gamma}$ maps $I$ into $I$ and $S(f)=f \circ \Psi$ is a continuous transformation of $B_{\gamma}(I)$ onto $B_{\alpha}(I)$ which preserves pointwise limits. This contradicts Theorem 5.2 and proves the theorem.

Dashiell and Lindenstrauss have proven that $B_{1}(I)$ and $B_{\alpha}(I)$ are not isomorphic [5]. It is not known, in general, whether $B_{\alpha}(I)$ and $B_{\gamma}(I)$ are isomorphic [4]. Addendum. Arnold Miller states that for each $a>2$ there is a model in which $P\left(E^{2}\right): \cdots M_{a}(R)$.

\section{References}

[1] R. H. Bing, Metrization of topological spaces, Canad. J. Math. 3 (1951), pp. 175--186.

[2] - W. W. Bledsoe and R. D. Mauldin, On generalized rectangles, Pacific J. Math. 51 (1974), pp. 27-36.

[3] D. D. Booth, Countably indexed ultrafilters, Thesis, Madison Wis. 1969.

[4] F. K. Dashie1l, Isomorphism problems for the Baire classes, Pacific J. Math. (to appear).

[5] - and J. Lindenstrauss, Some examples concerning strictly convex norms and $C(K)$ spaces, Israel J. Math. (to appear).

[6] N. Dunford and J. T. Schwartz, Linear Operators, Part I, New York 1967.

[7] F. Hausdorff, Set Theory, 2nd ed., New York 1962

[8] R.W. Heath, Screenability, pointwise paracompactness and metrization of Moore spaces, Canad. J, Math. 16 (1964), pp. 763-770.

[9] K. Kunen, Inaccessible properties of cardinals, Ph. D. Thesis, Department of Mathematics, Stanford University, August 1968.

[10] K. Kuratowski, Topology, Vol. I, Now York-London-Warszawa 1966.

[11] D. A. Martin and R. M. Solovay, Internal Cohen extensions, Ann. Math. Logic 2 (1970), pp. $143-178$.

[12] R. D. Mauldin, On the Baire System generated by a linear lattice of functions, Fund. Math. 68 (1970), pp. 51-59.

[13] - Baire functions, Borel sets and ordinary function systems, Advances in Math., April 1974.

[14] B. V. Rao, On discrete Borel spaces and projective sets, Bull. Amer. Math. Soc. 75 (1969), p. 614.

[15] - On discrete Borel spaces, Acta. Math. Acad. Scien. Hungaricae 22 (1971), p. 197.

[16] F. Rothberger, On some problems of Hausdorff and Sierpiniski, Fund. Math. 35 (1948), pp. $29-46$.

[17] - On families of real functions with a denumerable base, Ann. of Math. 45 (1,944), pp. 397-407.

[18] W. Sierpifiski, Sur tu probleme concernamt l's fonctions de première classe, Fund. Math. 27 (1936), pp, 191--200.

[19] E. Szpilrajn, The characteristic function of a sequence of sets and some of its applications, Fund. Math. 31 (1938), pp. 207-223.

[20] F. Tall, New results on the normal Moore space problem, Proc. of Washington State University Conference on General Topology, 1970, pp. 120-126.

[21] S. Ulam, Problem 74, Fund. Math. 30 (1938), p. 365.

[22] - Problems in Modern Mathematics, New York 1960

Reçu par la Rédaction le 8. 2. 1974

Accepté par la Rédaction le 14. 4. 1975 